The Astrophysical Journal, 512:140-144, 1999 February 10

(c) 1999. The American Astronomical Society. All rights reserved. Printed in U.S.A.

\title{
THE HOST GALAXY OF THE BROAD ABSORPTION LINE QSO PG 1700+518 AND ITS RING GALAXY COMPANION: NICMOS 1.6 MICRON IMAGING ${ }^{1}$
}

\author{
Dean C. Hines, Frank J. Low, and Rodger I. Thompson \\ AND \\ RAY J. WeYMANN AND LisA J. STORRIE-LOMBARDi \\ Carnegie Observatories, Pasadena, CA 91101; rjw@ociw.edu, lisa@ociw.edu \\ Received 1998 July 8; accepted 1998 September 16
}

Steward Observatory, University of Arizona, Tucson, AZ 85721; dhines@as.arizona.edu, flow@as.arizona.edu, thompson@as.arizona.edu

\begin{abstract}
The $1.6 \mu \mathrm{m}$ Near Infrared Camera and Multiobject Spectrometer image of the broad absorption line QSO PG $1700+518$ clearly resolves the QSO host galaxy and a ringlike companion. The companion is most likely a ring galaxy produced in a collision with the QSO galaxy $\sim 5 \times 10^{7}$ yr ago. The morphology of the PG $1700+518$ system is very similar to IRAS 04505-2958 (Boyce et al. 1996). Both objects were identified in a sample of eight QSOs selected by "warm" far-IR colors and extreme IR luminosities (Low et al. 1988). All eight QSOs show signs of strong interaction, and the presence of two head-on colliding systems in this sample may suggest that small impact parameters favor the energizing of IRluminous QSOs.
\end{abstract}

Subject headings: galaxies: peculiar — infrared: galaxies — quasars: individual (PG 1700+518)

\section{PG $1700+518$}

PG $1700+518\left(z_{\text {em }}=0.292\right)$ is one of the most infraredluminous (radio-quiet) QSOs known $\left[\log \left(L_{\mathrm{IR}} / L_{\odot}\right)=12.05\right.$; Low et al. 1988, 1989]. It was originally identified in the Palomar-Green Survey (Schmidt \& Green 1983) and subsequently found to be one of only $\approx 2 \%$ of QSOs that show broad absorption lines (BALs) from low-ionization species such as $\mathrm{Al}$ III $\lambda 1860$ and Mg II $\lambda 2800$ (Wampler 1985; Pettini \& Boksenberg 1985; Turnshek et al. 1985). Its IR luminosity, warm far-IR colors indicative of dust heated by a QSO UV continuum $\left[0.25 \leq F_{v}(25 \mu \mathrm{m}) / F_{v}(60 \mu \mathrm{m}) \leq 3\right.$ : Low et al. 1988, 1989], and its polarization properties (Hines \& Schmidt 1997; Schmidt \& Hines 1999; Schmidt, Hines, \& Smith 1997), all suggest a strong connection with the ultra- and hyperluminous infrared galaxies (Sanders et al. 1988a; Cutri et al. 1994; Hines et al. 1995).

Previous ground-based optical and near-IR images of PG $1700+518$ reveal a slight extension southwest and an arclike feature $\approx 2$ ".5 northeast of the bright point source (Hickson \& Hutchings 1987; Hutchings, Neff, \& Gower 1992; Stickel et al. 1995). The " $E+A$ " optical spectrum of the arc implies an old population of stars plus an $85 \mathrm{Myr}$ old starburst (Canalizo \& Stockton 1997; Stockton, Canalizo, \& Close 1998), and the small velocity offset between the arc and the QSO $\left(140 \mathrm{~km} \mathrm{~s}^{-1}\right)$ suggests that the two are physically associated. Recent ground-based images using adaptive optics have shown that the arc is a discrete companion galaxy (Stockton et al. 1998), but the detailed morphology of this structure is still difficult to determine from ground-based images. In this contribution we present a deep $1.6 \mu \mathrm{m}$ image obtained with the Near Infrared Camera and Multiobject Spectrometer (NICMOS) aboard the Hubble Space Telescope (HST).

\footnotetext{
${ }^{1}$ Based on observations with the NASA/ESA Hubble Space Telescope obtained at the Space Telescope Science Institute, which is operated by the Association of Universities for Research in Astronomy, Inc., under NASA contract NAS 5-26555.
}

In the next section we present details of our observations and data reductions that may be useful to other NICMOS programs. We present the observed properties of the companion and QSO host galaxy in $\S 3$, and we then briefly discuss the significance of these results for understanding the connection between ultra- and hyperluminous infrared galaxies and QSOs.

\section{OBSERVATIONS}

PG $1700+518$ was observed on UT 1997 October 28 through the F160W filter of NICMOS camera 2 (NIC2). A four-position spiral-dither pattern with 50.5 pixel offsets in the detector coordinate system was used to improve sampling and alleviate the effects of bad pixels, cosmic rays, some persistence, and other image artifacts. The half-integer offsets in four orthogonal directions ensure optimal sampling regardless of the initial placement of the object on the array. A fifth image was obtained at the initial dither position to fill the orbit.

The integrations were obtained at each dither position in MULTIACCUM mode with MIF512, NSAMP $=25$ $(514.39 \mathrm{~s})$, and reduced using CALNICA 3.0 (MacKenty et al. 1997). The DC offsets of the quadrants in individual frames were adjusted to eliminate discontinuities at the boundaries. In-flight flat-field observations were reduced with observed darks and applied. The frames were registered to within 0.1 pixel using bicubic interpolation and median combined. Conversion to a Johnson photometric system $\left(2.2 \times 10^{-6} \mathrm{Jy}^{\left.\mathrm{ADU}^{-1}\right)}\right.$ was based on the calibration analysis of the Instrument Definition Team (M. Rieke 1998, private communication). The final image has a $3 \sigma$ detection limit $\approx 5 \mu \mathrm{Jy}, H=20.8 \mathrm{mag} \operatorname{arcsec}^{-2}$, and a dynamic range of $\approx 60,000$.

A point-spread function (PSF) was constructed in a similar manner from observations of a field star obtained on UT 1997 October 26 with NIC2 F160W (courtesy of N. Scoville \& A. Evans 1997, private communication). These observations were also made using a four-position 
spiral dither, but with 20.5 pixel offsets, and were reduced as for PG $1700+518$. The vertical band in the final images of both PG $1700+518$ and the PSF star is an electronic artifact caused by saturation of the point source in the last few reads of each dither exposure (Thompson et al. 1998).

\section{RESULTS}

The final $1.6 \mu \mathrm{m}$ image of PG $1700+518$ is shown in Figure $1 a$. Although the QSO point source dominates the image, the arclike feature to the northeast (bottom of image) is clearly visible, and there is a suggestion of extended emission to the southwest.

The PSF star and PSF-subtracted image are shown in Figures $1 b$ and $1 c$, respectively. These show the $128 \times 128$ pixels centered on the QSO, where all the "dithered" images overlap. The PSF star and the point source (QSO) in PG $1700+518$ were aligned to within 0.1 pixels using bicubic interpolation. The final scaling was chosen to minimize the diffraction pattern while maintaining a monotonically increasing underlying "galaxy" flux in regions outside the innermost minimum in the Airy pattern. The PSF subtraction is rather noisy in regions where the minimum in Airy patterns of the object and PSF overlap (i.e., low signal noise), and it suffers from differences in the high spatial frequency structure of the PSF compared with the QSO. These differences are likely caused by slight focus changes induced by telescope breathing (see, e.g., Krist et al. 1998). Future observations of PSF stars observed at breathing offsets closer to those for PG $1700+518$, and simulations of the PSF using refined Tiny TIM models may provide improved PSF subtraction.

The arclike feature in Figure $1 c$ is resolved $(0,076$ pixel $^{-1}$ ) into a central condensation surrounded by a bright ring. The residual diffuse emission underlying the QSO point source and extending $\sim 2$ " southwest is presumably the QSO host galaxy. The measured $1.6 \mu \mathrm{m}$ flux of the entire system is equal to $6.3 \mathrm{mJy}( \pm 5 \%)$ and is in reasonable agreement with the ground-based (Johnson) $\mathrm{H}$-band flux equal to $7.2 \mathrm{mJy}( \pm 10 \%)$ measured by Low et al. (1989). The flux contributed by the QSO point source alone is $\approx 5.8 \mathrm{mJy}$, so about $90 \%$ of the total $1.6 \mu \mathrm{m}$ flux emanates from the unresolved QSO nucleus.

\subsection{The Companion: A Ring Galaxy}

The PSF subtraction leaves some residual artifacts superimposed on the QSO host galaxy, so we first describe those features of Figure $1 c$ that are not obviously associated with the QSO host galaxy (i.e., the companion galaxy). The morphology of the companion is strikingly similar to that of collisional ring galaxies (Lynds \& Toomre 1976, and the review by Appleton \& Struck-Marcell 1996), and in particular to the "banana-type" morphology of WN 1 (Wakamatsu \& Nishida 1987; Appleton \& Marston 1997), which is thought to be caused by a slightly off-center collision (Appleton \& Struck-Marcell 1987). The secondary condensation reported by Stockton et al. (1998, their knot b) is found to be an elongated brightness enhancement within the ring.

Table 1 compares the companion with other collisional ring galaxies from the survey by Appleton \& Marston (1997). It is difficult to completely isolate the ring from the QSO host galaxy, so we have measured only the half that is clearly uncontaminated by the QSO host, and we excluded the nucleus of the ring galaxy. We then multiplied by 2 to estimate the total ring flux $(235 \pm 30 \mu \mathrm{Jy})$ and added back the contribution from the ring galaxy nucleus $(23 \pm 5 \mu \mathrm{Jy})$ to obtain the total flux from the companion galaxy $\left(258 \pm 30 \mu \mathrm{Jy} ; m_{\mathrm{H}}=16.6\right)$. The errors were estimated from the range of fluxes obtained using slightly different (square) aperture sizes and centers. For other wavelength bands, we rely on the values obtained for PG $1700+518$ by Stickel et al. (1995) and Canalizo \& Stockton (1997). The linear diameter of the ring $(\sim 12.6 \mathrm{kpc})$, the $R-H(V-J$ rest-frame $)$ colors of the ring plus bulge, and the ratio $L_{V} / L_{H}$ are typical of values observed for ring galaxies locally.

The $E+A$ optical spectrum of the bulge-plus-ring implies an old population of stars plus an $85 \mathrm{Myr}$ old starburst (Canalizo \& Stockton 1997; Stockton et al. 1998), which is consistent with the bursts of star formation observed within the rings of ring galaxies. The small velocity offset between the companion and the QSO $\left(140 \mathrm{~km} \mathrm{~s}^{-1}\right)$ is also similar to that seen between the colliding galaxies in other ring systems (see, e.g., WN 1; Wakamatsu \& Nishida 1987) and suggests that $\sim 5 \times 10^{7}$ yr have passed in the rest frame since the companion and the QSO passed each other,

TABLE 1

Properties of Local RING GALAXIES ${ }^{\mathrm{a}}$ AND PG $1700+518 \mathrm{R}$

\begin{tabular}{|c|c|c|c|c|c|c|}
\hline Name & $z$ & $\begin{array}{l}\text { Diameter } \\
\quad(\mathrm{kpc})\end{array}$ & $\begin{array}{l}V-J \\
(\mathrm{mag})\end{array}$ & $\begin{array}{c}L_{V}^{\mathrm{b}} \\
\left(10^{42} \mathrm{ergs} \mathrm{s}^{-1} \mathrm{~cm}^{-2}\right)\end{array}$ & $\begin{array}{c}L_{H}^{\mathrm{b}} \\
\left(10^{42} \operatorname{ergs~s}^{-1} \mathrm{~cm}^{-2}\right)\end{array}$ & $L_{V} / L_{H}$ \\
\hline LT $41 \ldots \ldots \ldots \ldots \ldots$ & 0.074 & 26.7 & 3.17 & 100.8 & 2.1 & 48.6 \\
\hline Cartwheel .............. & 0.030 & 41.4 & 2.45 & 140.6 & 1.8 & 77.1 \\
\hline III Zw $33 \ldots \ldots \ldots \ldots$ & 0.027 & 12.6 & 2.16 & 52.5 & 0.6 & 95.3 \\
\hline NGC $985 \ldots \ldots \ldots \ldots$ & 0.044 & 76.9 & 3.40 & 340.2 & 13.5 & 25.3 \\
\hline II Zw $28 \ldots \ldots \ldots \ldots . . . .$. & 0.029 & 11.7 & 2.01 & 39.3 & 0.4 & 113.5 \\
\hline II $\mathrm{Hz} 4 \ldots \ldots \ldots \ldots \ldots$ & 0.043 & 27.0 & 2.78 & 148.3 & 2.2 & 67.1 \\
\hline WN $1 \ldots \ldots \ldots \ldots \ldots$ & 0.056 & 27.7 & 3.64 & 163.5 & 5.0 & 32.4 \\
\hline VII Zw $466 \ldots \ldots \ldots \ldots$ & 0.049 & 26.5 & 2.57 & 90.0 & 1.1 & 79.3 \\
\hline Abell 76 .............. & 0.011 & 4.5 & 1.79 & 7.4 & 0.1 & 144.2 \\
\hline LT $36 \ldots \ldots \ldots \ldots \ldots$ & 0.027 & 9.4 & 2.89 & 56.5 & 0.9 & 59.6 \\
\hline Mean $(1 \sigma) \ldots \ldots \ldots \ldots$ & & $26(21)$ & $2.7(0.6)$ & $114(94)$ & $3(4)$ & $74(37)$ \\
\hline PG $1700+518 R \ldots \ldots$ & 0.292 & 12.6 & $2.4^{\mathrm{c}}$ & $199.6^{\mathrm{d}}$ & 2.8 & 71.6 \\
\hline
\end{tabular}

${ }^{a}$ After Appleton \& Marston 1997.

${ }^{\mathrm{b}} H_{0}=75 \mathrm{~km} \mathrm{~s}^{-1} \mathrm{Mpc}^{-1}$ and $q_{0}=0.1$.

${ }^{c}$ Corrected to rest frame from the observed $R$ band (Stickel et al. 1995) and our NICMOS $1.6 \mu \mathrm{m}$ fluxes assuming

$F_{v}$ (rest) $\sim(1+z)^{\alpha_{v}}, F_{v}(\mathrm{obs})$, and $F_{v} \propto v^{\alpha_{v}}, \alpha_{v}=-1.0$ (Low et al. 1989).

${ }^{d}$ Estimated from the optical spectrum presented by Canalizo \& Stockton 1997. 

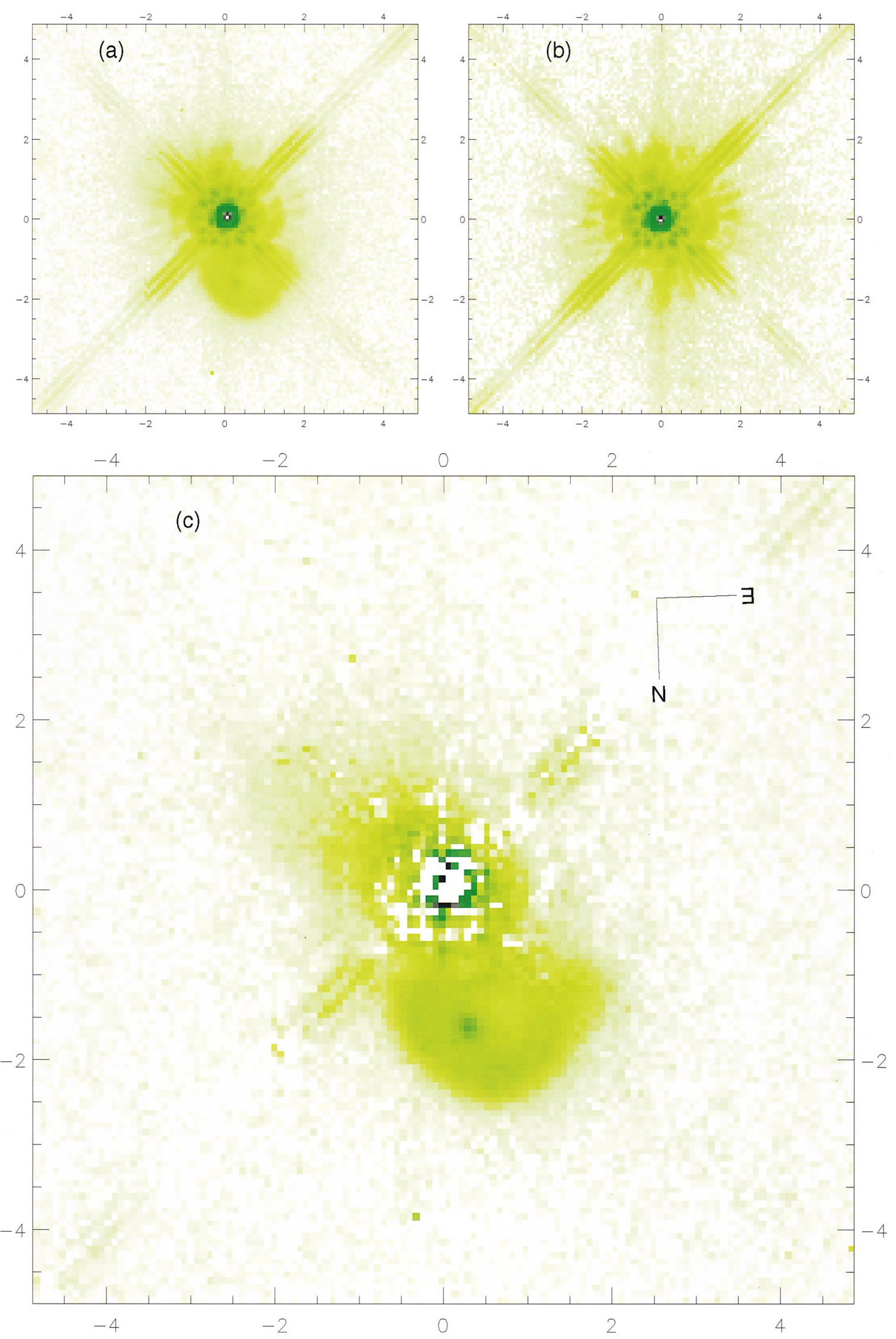

FIG. 1.-NICMOS $1.6 \mu \mathrm{m}$ imaging of PG $1700+518$. (a) The observed background-subtracted image; (b) the background-subtracted and scaled PSF image; $(c)$ the PSF-subtracted image (expanded for clarity). The images are 9".6 on a side (i.e., $128 \times 128$ pixels) and are displayed with a log stretch to emphasize the low surface brightness features. The PSF has been scaled to minimize the residual diffraction structure without causing a large negative "dip" in the QSO host galaxy (see text). The $3 \sigma$ detection limit is equal to $20.8 \mathrm{mag} \operatorname{arcsec}^{-2}$, and the dynamic range is $\approx 60,000$. The images are shown as observed; the HST FITS header keyword is ORIENT = 177.6 (i.e., north is down, east is right). 
assuming projection factors of order $0.5 .^{2}$ This age is consistent with the age of the starburst inferred from the optical spectrum (Stockton et al. 1998).

The evidence implies that the companion to $P G$ $1700+518$ (hereafter PG $1700+518 \mathrm{R}$ ) is a classical, collisionally produced ring galaxy. The similarity between the inferred age of the starburst in the ring and the travel time between the QSO and companion suggests that PG $1700+518 \mathrm{R}$ was produced by a near head-on collision between the QSO host galaxy and PG $1700+518$ R's progenitor.

\subsection{The QSO Host}

Diffuse emission surrounding the QSO extends to $\sim 5^{\prime \prime}$ in deep (3 $\sigma=25 \mathrm{mag} \operatorname{arcsec}^{-2}$ ), ground-based $V$-band images (Hickson \& Hutchings 1987; Hutchings et al. 1992). This structure is presumably associated with the QSO host galaxy, and not the ring galaxy, given the sharpness of the ring edge seen in our images (Fig. 1c) and the fact that the near-IR and optical morphologies of ring galaxies are nearly identical (Appleton \& Marston 1997).

The southwestern extended emission seen in Figure $1 c$ is also present in the ground-based optical and near-IR images (Hutchings et al. 1992; Stickel et al. 1995). It is probably associated with the QSO host and may be tidal debris produced by the collision that formed the ring galaxy. There is also an apparent extension under and east of the northeastern diffraction spike. While this feature might possibly be associated with the ring galaxy, it is probably part of the QSO host. If it is tidal debris, it may have a different color (i.e., redder) than the ring.

Residuals from the PSF subtraction mask some of the structure within 1 " and along the diffraction spikes, but the morphology of the QSO host galaxy is clearly not elliptical. Instead it more closely resembles the disturbed structures seen in other infrared ultraluminous galaxies (see, e.g., Hutchings \& Neff 1988; Sanders et al. 1988b; Surace et al. 1998). Subtracting the flux contributed by PG $1700+518 \mathrm{R}$ and by the QSO point source yields a total flux for the QSO host galaxy alone, $\sim 0.3 \mathrm{mJy}(H \sim 16.5)$, consistent with the $18.8 \lesssim H \lesssim 15.6$ inferred by Stickel et al. (1995). Assuming a $V-H$ color typical of spiral galaxies (with appropriate $K$ correction) yields $L \sim 2 L^{*}$ for the QSO host galaxy, typical of luminous low-redshift QSOs (see, e.g., McLeod \& Rieke 1994).

The double radio source associated with PG $1700+518$ (Neff \& Hutchings 1992; Hutchings et al. 1992) is apparently not directly associated with the QSO host galaxy or PG $1700+518 \mathrm{R}$, since the position angle differs by $\sim 50^{\circ}$.

\section{DISCUSSION}

It has been suggested that at least some QSOs undergo an evolutionary sequence in which the collision of two

\footnotetext{
${ }^{2}$ This velocity difference also suggests that the ring galaxy lies behind the QSO and is therefore not the current source of the BAL material.
}

massive, gas-rich spiral galaxies produces vigorous star formation and energizes an active nucleus that eventually "burns" a hole in its dusty cocoon to emerge as a typical UV-excess QSO (see, e.g., Sanders et al. 1988a; Sanders \& Mirabel 1996, and references therein). Along this evolutionary path, BAL features like those in PG $1700+518$ may be produced by material that is expelled by the emerging QSO.

The PG $1700+518$ system shows a marked similarity to the IRAS-discovered infrared-luminous QSO IRAS 04505-2958 (Low et al. 1988), which itself consists of a QSO, a host galaxy, and a ringlike companion (Boyce et al. 1996). The total non-QSO light in the PG $1700+518$ system $\sim 4 L^{*}$ is comparable to that obtained for IRAS 04505-2958 (6L*; Boyce et al. 1996). The large host and companion luminosities, the ringlike morphologies of their companions, and their dusty environments all support the hypothesis that both of these systems resulted from collisions of massive spiral galaxies.

PG $1700+518$ appears among eight radio-quiet QSOs selected by their "warm" far-IR colors from the IRAS point-source catalog (Low et al. 1988). All eight QSOs appear to be strongly interacting (Hutchings \& Neff 1988; Hutchings \& Morris 1995; Boyce et al. 1996; Surace et al. 1998; Hines et al. 1999), and two are associated with ringlike companions. Ultraluminous infrared galaxies with cooler far-IR colors more typical of vigorous star formation also appear to be in interacting systems, but none have so far been found to be associated with collisional ring galaxies. Perhaps the energizing of an IR-luminous QSO is favored by interactions with small-impact parameters like the near head-on collisions necessary to produce ring galaxies.

At $z=0.292$, PG $1700+518 \mathrm{R}$ is one of the most distant ring galaxies known. Observed at a higher redshift, the ring morphology would still be evident even though other morphological indicators of interaction such as tidal tails might be difficult to detect. Therefore, ring galaxies provide an important marker for identifying galaxy collisions at high redshifts (see, e.g., Lavery et al. 1996). If ring galaxies are commonly associated with IR-luminous QSOs, as suggested herein, then future surveys for IR-luminous QSOs with instruments such as those aboard SIRTF may uncover even higher redshift examples of the collisional ring galaxy phenomenon.

We thank G. Schneider and D. Lytle for suggestions regarding PSF subtraction. We are grateful to $M$. Rieke and E. Stobie for assistance with the darks and flat fields. It is a pleasure to thank A. Stockton, G. Canalizo, and L. Close for providing details of their adaptive optics observations prior to publication. Thanks to G. Rieke and A. Quillen for valuable discussions. Finally, we thank Z. Levay at STScI for his assistance with understanding color lookup tables in Adobe Photoshop. This work is supported by NASA grant NAG 5-3042 to the NICMOS instrument definition team.

\section{REFERENCES}

Appleton, P. N., \& Marston, A. P. 1997, AJ, 113, 201

Appleton, P. N., \& Struck-Marcell, C. 1996, Fundam. Cosmic Phys., 16, 111

1987, ApJ, 318, 103

Boyce, P. J., et al. 1996, ApJ, 473, 760

Canalizo, G., \& Stockton, A. 1997, ApJ, 480, L5

Cutri, R. M., Huchra, J. P., Low, F. J., Brown, R. L., \& Vanden Bout, P. A.

1994, ApJ, 424, L65
Hines, D. C., Low, F. J., Evans, A. S., Scoville, N. Z., Rieke, M., Thompson, R. I., \& Schneider, G. 1999, in preparation

Hines, D. C., \& Schmidt, G. D. 1997, in ASP Conf. Ser. 128, Mass Ejection from Active Galactic Nuclei, ed. N. Arav, I. Shlosman, \& R. J. Weymann (San Francisco: ASP), 59

Hines, D. C., Schmidt, G. D., Smith, P. S., Cutri, R. M., \& Low, F. J. 1995, ApJ, 450, L1

Hickson, P., \& Hutchings, J. B. 1987, ApJ, 312, 518 
Hutchings, J. B., \& Morris, S.C. 1995, AJ, 109, 1541

Hutchings, J. B., \& Neff, S. G. 1988, AJ, 109, 1541

Hutchings, J. B., Neff, S. G., \& Gower, A. C. 1992, PASP, 104, 62

Krist, J. E., Golimowski, D. A., Schroeder, D. J., \& Henry, T. J. 1998, PASP, 110, 1046

Krist \& Hook 1997

Lavery, R. J., Seitzer, P., Suntzeff, N. B., Walker, A. R., \& Da Costa, G. S. 1996, ApJ, 467, L1

Low, F. J., Cutri, R. M., Kleinmann, S. G., \& Huchra, J. P. 1989, ApJ, 340, L1

Low, F. J., Huchra, J. P., Kleinmann, S. G., \& Cutri, R. M. 1988, ApJ, 327, L41

Lynds, C. R., \& Toomre, A. 1976, ApJ, 209, 382

MacKenty, J. W., et al. 1997, NICMOS Instrument Handbook (v. 2.0; Baltimore: STScI)

McLeod, K. K., \& Rieke, G. H. 1994, ApJ, 431, 137

Neff, S. G., \& Hutchings, S. G. 1992, AJ, 103, 1746

Pettini, M., \& Boksenberg, A. 1985, ApJ, 294, L73

Sanders, D. B., \& Mirabel, I. F. 1996, ARA\&A, 34, 749
Sanders, D. B., Soifer, B. T., Elias, J., Madore, B., Matthews, K., Neugebauer, G., \& Scoville, N. 1988a, ApJ, 325, 74

Sanders, D. B., Soifer, B. T., Elias, J. H., Neugebauer, G., \& Matthews, K. 1988b, ApJ, 328, L35

Schmidt, G. D., \& Hines, D. C. 1999 , ApJ, submitted

Schmidt, G. D., Hines, D. C., \& Smith, P. S. 1997, in ASP Conf. Ser. 128, Mass Ejection from Active Galactic Nuclei, ed. N. Arav, I. Shlosman, \& R. J. Weymann (San Francisco: ASP), 106

Schmidt, M., \& Green, R. F. 1983, ApJ, 269, 352

Stickel, M., Fried, J. W., McLeod, K. K., \& Rieke, G. H. 1995, AJ, 109, 1979

Stockton, A., Canalizo, G., \& Close, L. M. 1998, ApJ, 500, L121

Surace, J. A., Sanders, D. B., Vacca, W. D., Veilleux, S., \& Mazzarella, J. M. 1998, ApJ, 492, 116

Thompson, R. I., Rieke, M., Schneider, G., Hines, D. C., \& Corbin, M. R. 1998, ApJ, 492, L95

Turnshek, D. A., Foltz, C. B., Weymann, R. J., Lupie, O. L., McMahon, R.G., \& Peterson, B. M. 1985, ApJ, 294, L1

Wampler, E. J. 1985, ApJ, 296, 416

Wakamatsu, K., \& Nishida, M. T. 1987, ApJ, 315, L2 